\title{
Demographic Characteristics Related to Motor Skills in Children Aged 5-7 Years Old
}

\author{
You Fu' ${ }^{1 *}$, Ryan D. Burns ${ }^{2}$ \\ ${ }^{1}$ School of Community Health Sciences, University of Nevada, Reno, 1664 N Virginia Street, Reno, NV 89557 \\ ${ }^{2}$ Department of Health, Kinesiology, and Recreation, University of Utah, 250 S 1850 E, Salt Lake City, UT 84112
}

Corresponding Author: You Fu, E-mail: youf@unr.edu

\section{ARTICLE INFO}

\section{Article history}

Received: November 2, 2017

Accepted: February 15, 2018

Published: April 30, 2018

Volume: 6 Issue: 2

Conflicts of interest: None

Funding: None

\begin{abstract}
Background: Motor skill is important to young children's overall well-being. However, there has been a paucity of work examining the demographic characteristics on young children' motor skill. Objective: The purpose of this study was to examine the differences in motor skills across socio-economic status (SES) and grade levels in elementary school children. Method: Participants were 651 kindergarten to $2^{\text {nd }}$ grade children (mean age $=6.2 \pm 0.9$ years; 305 girls, 346 boys) recruited from two low SES schools and another two high SES schools. Selected motor skill items were measured using the Test for Gross Motor Development$3^{\text {rd }}$ Edition (TGMD-3) instrument. Data were collected once at each school during physical education class and recess period. A $4 \times 3 \times 2 \times 2$ Multivariate Analysis of Variance (MANOVA) test was employed to examine the differences among grade, SES, ethnicity, and sex on TGMD3 scores. Results: There were significant main effects for grade (Wilks' lambda $=0.34$, $\mathrm{F}(2,1274)=229.6, p<0.001)$ and SES (Wilks' lambda $=0.70, \mathrm{~F}(2,637)=136.3, p<0.001)$. Follow-up tests revealed statistically significant differences between grades on locomotor, object control and overall TGMD-3, with the $2^{\text {nd }}$ graders displaying highest mean scores, followed by $1^{\text {st }}$ graders and kindergarteners. Follow-up tests suggested that high SES children displaying statistically significant higher mean scores than low SES students on all motor skill variables. Conclusion: Older children demonstrated higher motor competence levels, and those with high SES displayed higher motor skill levels than lower SES children.
\end{abstract}

Key words: Motor Skills, Exercise, Child, Social Class

\section{INTRODUCTION}

Motor skills are the basic human movements that are commonly identified by locomotor skills (e.g. running, jumping, sliding, etc.) and manipulative skills (e.g. kicking, throwing, or catching, etc.) (Burton \& Miller, 1998; Pangrazi \& Beighle, 2013; Barnett, Ridgers, \& Salmon, 2015). The development of motor skills is a continuous and age-related process of change in movement. As age progresses, motor skills proceed from simple to complex locomotor and manipulative movements, which function as the building blocks for more advanced skills (Burton \& Miller, 1998; Payne \& Isaacs, 2011). According to Burton and Miller's (1998) movement skill assessment model, motor skills facilitate young aged individuals control bodies, adopt surrounding environments, achieve complex tasks that are involved in athletic and daily life activities (Davis \& Burton, 1991). Therefore, an optimal development of young children's motor skills is of significant importance for their healthy physical and social conditions, sport performance, and general daily living activities (Deflandre, Lorant, Gavarry, \& Falgairette, 2001; Williams et al., 2008; Trudeau \& Shephard, 2008; Lai et al., 2014; Robinson et al., 2015; Burns, Brusseau, Fu, \& Hannon, 2017).
Although an optimal development of these skills is important for the growing child, many children are still unable to achieve motor skill competence (Robinson et al., 2015). In fact, children who demonstrated low level of motor skills may also display low level of motivation, such as perceived physical competence and self-efficacy (Robinson, Rudisill, \& Goodway, 2009; Barnett et al., 2015). The lack of motivation compromises physical activity participation and health-related fitness, and thus can exacerbate health risk if low levels of these constructs track through adolescence and into adulthood (Stodden, Goodway, \& Langendorfer, 2008; Burns, Brusseau, Fu, \& Hannon, 2015; Ali, Pigou, Clarke, \& McLachlan, 2017).

Previous research has suggested that incompetency in motor skills was associated with lower levels of physical activity behaviors (Hardy, et al., 2012; Logan, Webster, Getchell, Pfeiffer, \& Robinson, 2015). In addition, a recent research reported a significant correlation between children's motor skills and their cardio-metabolic risk that was mediated through aerobic fitness (Burns, Brusseau, Fu, \& Hannon, 2017). Although it is beneficial for all students to develop motor skills in school settings, children with low 
socio-economic status (SES), or those from low-income families, may have low motor skill levels. This is because of fewer opportunities to participate in sports and recreational activities before and after school, and the limited physical education time during school (Lampard, Jurkowski, Lawson, \& Davison, 2013). Particularly, it has been documented that low-income Hispanic and African American preschool children had a delayed proficiency in motor skills (Goodway \& Branta, 2003; Goodway \& Rudisill, 1997).

Compared to children from middle and high-income households, children from low-income families may be at risk for poor motor skill development due to the limited access to physical activity and sport participation outside of school settings. These children may also have limited resources to safe playground areas or equipment for motor skill competence (Kercood et al., 2015; Eime, et al., 2017). Despite these positive findings in disadvantaged preschool children, there has been a paucity of work examining the demographic factors relating to motor skill levels in young elementary school-aged children with the consideration that young individual's motor skill development is a continuous process and changes rapidly during the early years of age. Due to the importance of developing motor skills, the purpose of this study was to examine the differences in motor skills among SES and grades in a sample of young elementary school-aged children. It was hypothesized that older grade cohorts will display greater TGMD-3 scores compared to younger grade cohorts. We also hypothesized that high SES children will demonstrate higher levels on both locomotor and ball skills compared to children from lower income schools.

\section{MATERIALS AND METHODS}

\section{Participants}

A convenience sample of 651 kindergarten through $2^{\text {nd }}$ grade children (mean age $=6.2 \pm 0.9$ years; 305 girls, 346 boys) were recruited from four urban elementary schools located in the Western U.S. There were two high SES schools in the middle and high-income household areas. All schools were within the same school district. In the high SES sample (141 girls, 172 boys), approximately $43.9 \%$ of the children were of Caucasian ethnicity, 24.4\% were Hispanic/Latino, 16.3\% were African American, 10.9\% were Asian, and 4.5\% were classified as other. In the low SES sample (164 girls, 174 boys), approximately $14.2 \%$ of the children were of Caucasian ethnicity, $45.0 \%$ were Hispanic/Latino, $27.5 \%$ were African American, 5.3\% were Asian, 8.0\% were classified as other. Children were recruited in this research only if they met the inclusion criteria: (1) aged 5 - 7 years; and (2) not diagnosed with physical and/or mental disability according to school records. Written assent was obtained from the children and consent was obtained from the parents prior to data collection. The University Institutional Review Board approved the study protocols.

\section{Study Design}

Children in the high SES schools were scheduled to have 2-3 physical education classes per week taught by certified physical educators, in addition to recess opportunities for physical activity. The two low SES schools were both "Nevada Zoom" schools that received government financial assistance. "Nevada Zoom" schools are given supplemental government funding for tutoring, smaller class sizes, and extended learning opportunities. This additional funding has the purpose to accelerate learning for students where English is not the primary language. There was no physical education at these schools, but children participated into physical activities during multiple recesses in every school day.

\section{Instrumentation and Tools}

The Test for Gross Motor Development Edition-3 (TGMD-3) was used to assess children's motor skills (Webster \& U1rich, 2017). The TGMD-3, upgraded from TGMD-2 (Ulrich, 2000 ), is a validated assessment battery of gross motor skills for children 3-10 years old. The TGMD-3 assessed motor skills across 13 movement skills within locomotor and object control subtests, respectively. The locomotor sub-tests comprised run, skip, slide, gallop, hop, and horizontal jump. The object control sub-tests included the overhand throw, underhand throw, catch, dribble, kick, one-hand strike, and two-hand strike. The locomotor and object control subtest scores were 46 and 54 respectively, and the total TGMD-3 scores were 100. Each child in this study performed the test items across two trials that were individually scored using specific performance criteria $(0=$ did not perform correctly; $1=$ performed.

\section{Procedures}

Gross motor skills were measured once at each school in the order of two low SES schools first, followed two high SES schools. Upon the entrance to the gym, a typical class of students was divided into two stations, which comprised of locomotor sub-tests and object control sub-tests with one research assistant supervised each station. Students switched over stations after completing all their sub-tests. For each sub-test, the research assistants demonstrated the movement before collecting data then scored using the protocols outlined in Webster \& Ulrich (2017). Two trained research assistants scored all TGMD-3 data live in these four schools during physical education classes or recess in the spring semester. One research assistant scored locomotor sub-tests at all schools and the other research assistant scored object control sub-tests at all schools to maintain testing consistency. Throughout the entire data collection period, the two research assistants were not aware of the SES difference among schools, which may increase the internal validity of the results and reduces the potential for bias.

Research assistants were trained for one week prior to the commencement of data collection. Training included a seminar tutoring the TGMD-3 guideline and scoring protocol. Two research assistants also practiced coding TGMD-3 among a sample of elementary school-aged children from a difference school for two sessions, with the purpose to calculate inter- and intra-scorer agreement using live and video coding. Each research assistant scored both locomotor and 
object control sub-tests. The inter-observer agreement (the agreement of coefficient between different observers) was 0.90 . The intra-observer agreement (the agreement of coefficient within the same observer over time) was 0.91 for the first observer and 0.93 for the second observer.

\section{Statistical Analysis}

Data were checked for Gaussian distributions using k-density plots and screened for outliers using z-scores $( \pm 3.0 \mathrm{z}$-score cut-point). To determine the eligibility to use a multivariate model, bivariate associations among the observed variables were examined using Pearson product-moment correlations. All of the correlations among the dependent variables were statistically significant, being moderate-to-strong in magnitude. Therefore, a $4 \times 3 \times 2 \times 2$ Multivariate Analysis of Variance (MANOVA) test was used to examine the differences among ethnicity (White, Black, Asian, Other), grade level (Kindergarten, $1^{\text {st }}$ grade, $2^{\text {nd }}$ grade), sex (girl, boy), and SES (high, low) on the gross motor skill variables. Both main effects and interactions were examined within the multivariate model. The dependent variables were the locomotor subtest score, the ball skill subtest score, and the TGMD-3 total score. If a statistically significant omnibus multivariate model was found using Wilks' lambda, follow-up univariate tests were explored with Bonferroni post hoc tests. Pair-wise comparison effect sizes were calculated using Cohen's delta (d), in that $\mathrm{d}<0.20$ indicting a small effect size, $\mathrm{d}=0.50$ indicating a medium effect size, and a $\mathrm{d}>0.80$ indicating a large effect size (Cohen, 1988). Alpha level was set a $p \leq 0.05$ and the analysis was conducted using SPSS 25.0 statistical software package (IBM Inc., Armonk, NY, USA).

\section{RESULTS}

The descriptive data for the total sample and within sex groups was presented in Table 1. Pearson correlations among all motor skill observed variables (individual items and subtest scores) were presented in Table 2 . There were statistically significant positive and weak-to-strong ( $\mathrm{r}=0.13$ to 0.88 ) correlations among the TGMD-3 subtests scores and total scores $(p<0.01)$, warranting multi-variate analysis.

The omnibus MANOVA model yielded two statistically significant main effects for grade (Wilks' lamb$\mathrm{da}=0.34, \mathrm{~F}(2,1274)=229.6, p<0.001)$ and SES (Wilks' lambda $=0.70, \mathrm{~F}(2,637)=136.3, p<0.001)$. There is no other statistically significant multivariate main effects or interactions. Assumptions of MANOVA were confirmed via approximately univariate Gaussian distributions across all dependent variables (multivariate normality), no clustering within the data structure (independence of observations), adequate sample size achieving at least $80 \%$ power (a priori), a lack of extreme multicolinarity among the dependent variables $(r<0.90)$, and a non-significant Box's M test supporting homogeneity of variance-covariance matrices. Grade and SES mean differences on gross motor skill are presented in Figures 1 and 2, respectively. The follow-up Bonferroni post-hoc tests showed that there were statistically significant differences on locomotor sub-

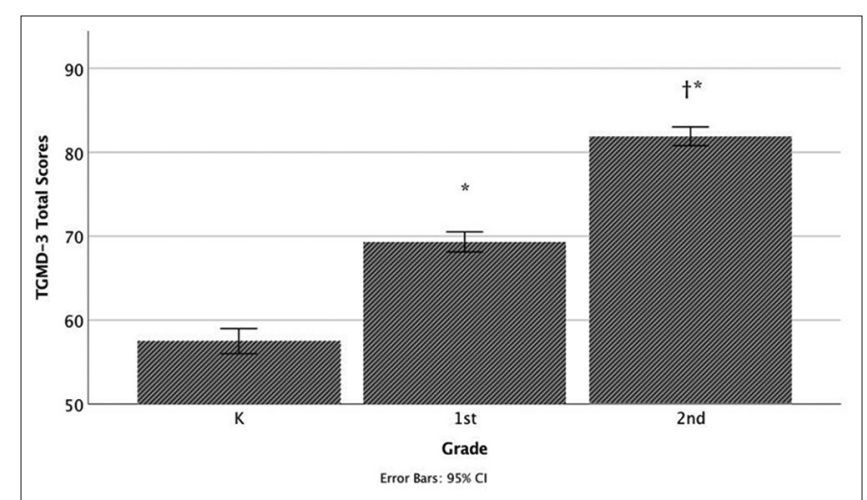

Figure 1. TGMD-3 scores across observed grades levels.

* indicates statistical significance, $\mathrm{p}<0.05$; $\uparrow$ indicates statistical significance, $\mathrm{p}<0.01$; Error bars are standard deviations

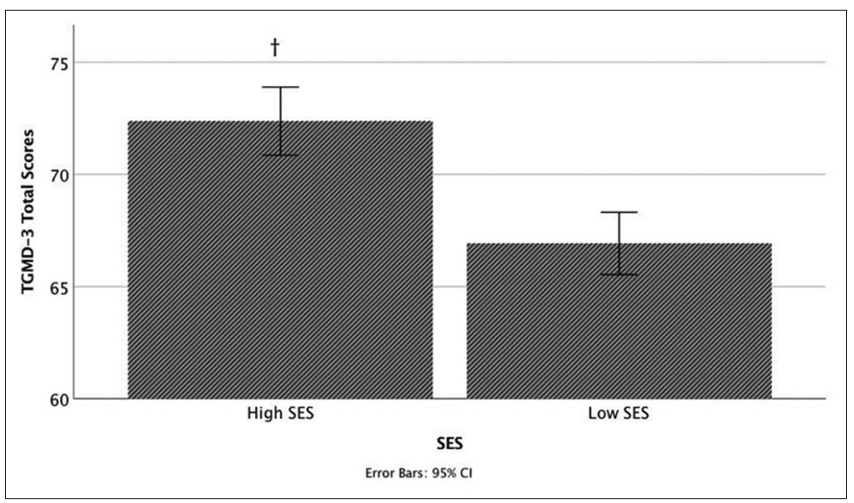

Figure 2. TGMD-3 scores between SES groups. $\uparrow$ indicates statistical significance, $\mathrm{p}<0.05$; Error bars are standard deviations

test scores across grades. Specifically, the $2^{\text {nd }}$ graders displayed higher mean scores compared to the $1^{\text {st }}$ graders (mean difference $=4.4, p<0.001, \mathrm{~d}=0.91$ ), the $1^{\text {st }}$ graders displayed higher mean scores compared to the kindergarteners (mean differences $=4.30, p<0.001, \mathrm{~d}=0.68$ ), and $2^{\text {nd }}$ graders displaying higher mean scores compared to kindergarteners (mean difference $=8.65, p<0.001, \mathrm{~d}=1.11$ ) With respect to the ball skill subtest scores, the $2^{\text {nd }}$ graders displayed higher mean scores than the $1^{\text {st }}$ graders (mean differences $=8.19, p<0.001, \mathrm{~d}=1.23)$; the $1^{\text {st }}$ graders displayed higher mean scores than the kindergarteners (mean difference $=7.55, p<0.001, \mathrm{~d}=1.14$ ), and the $2^{\text {nd }}$ graders displayed higher mean scores than the kindergarteners (mean difference $=15.74, p<0.001, \mathrm{~d}=2.38$ ). In terms of the TGMD-3 total scores, the $2^{\text {nd }}$ graders displayed higher mean scores as compared to $1^{\text {st }}$ graders (mean difference $=$ $12.57, \mathrm{p}<0.001, \mathrm{~d}=1.14), 1^{\text {st }}$ graders displayed higher mean scores as compared to kindergarteners (mean difference $=$ $11.82, \mathrm{p}<0.001, \mathrm{~d}=1.07)$, and $2^{\text {nd }}$ graders displayed higher mean scores as compared to kindergarteners (mean difference $=24.38, p<0.001, \mathrm{~d}=2.22$ ) .

The follow-up Bonferroni post hoc tests also suggested that there were statistically significant differences between SES levels, with the high SES students displaying statistically significant higher mean scores compared to low SES students on locomotor sub-test scores (mean difference $=1.09$, $p<0.001, \mathrm{~d}=0.17$ ), object control sub-test scores (mean 
difference $=2.56, p<0.001, \mathrm{~d}=0.31$, and TGMD-3 total scores (mean difference $=5.45, p<0.001, \mathrm{~d}=0.42$ ).

\section{DISCUSSION}

Although there is a growing body of evidence, the work examining the demographic characteristics on young children's motor skills has not yet been explored in depth. Accordingly, the purpose of this cross-sectional study was to investigate the demographic characteristics on motor skills in a sample of aged 5-7 years old children recruited from four rural schools. One of the salient findings was that the development of children's motor skills improved with the progression of age, as older children in the sample demonstrated statistically significant higher TGMD-3 sub-tests and overall scores than the younger participants. Another salient finding was that children with high SES had a significantly higher motor skill level compared to low SES children, or those from low-income families.

In the present study, the $2^{\text {nd }}$ grade students achieved statistically higher mean scores than the $1^{\text {st }}$ grade students on locomotor, object control, and overall TGMD-3, and a similar finding was further detected between the $1^{\text {st }}$ grade students and kindergarteners. These results demonstrated that there was a positive relationship between the development of motor skill and gerontology. Based on the theoretical perspectives in motor development, maturational theory of child development (Gesell, 1928) explained young children's motor developmental changes as the function of maturational processes, particularly, through the central nervous systems, which control the motor development. The maturational theory of child development further claimed that children's motor development is an internal growth driven by biological development (Gesell, 1928). The theoretical perspectives were soundly echoed by the findings of the present study, in that older students in the sample who characterized with higher level of biological and physiological development demonstrated higher levels of the motor skills development.

Present results were also in line with another research (Martinek et al., 1978) that aimed to compare the race and age differences on elementary school students' physical activity and motor skills. The study reported a progressive improvement in motor skill scores across grades, in specific, the motor skill mean scores for Grade 1, 2 and 3 were $27.30,30.09$, and 37.62 , respectively. The findings of this study strengthened the contention that the process of maturation has a definite effect on motor performance in young children. It has also been suggested that motor skills and physical activity were significant associated with each other (Fisher at al., 2005; Stodden at el., 2008; Holfelder \& Schott, 2014; Barnett et al., 2015). Therefore, a better understanding on the development of gross motor skills across various school grades may further lead to increased physical activity participation and health-related fitness levels, as this cohort tracks through adolescence and into adulthood (Stodden, Gao, Goodway, \& Langendorfer, 2014).

We also found high SES sample in this study achieved statistically higher scores on locomotor, object control, and overall TGMD-3 as compared to the low SES children. This finding is in line with our hypothesis and is partially supported by theoretical perspectives as well as previous studies. Gesell (1928) claimed that children's social environment plays an important role in their motor skills development, and these external incentives were most effective when they were synchronized with the inner maturational timeline. Stodden et al. (2008) suggested that young children's different experiences might have attributed to the various lev-

Table 1. Descriptive statistics for the total sample and within each sex group (means and standard deviations)

\begin{tabular}{lccc}
\hline & Total & Girls & Boys \\
$(\mathbf{N}=\mathbf{6 5 1})$ & $(\mathbf{n = 3 0 5})$ & $3.2(0.7)$ \\
\hline Run (Raw score 0-4) & $3.2(0.7)$ & $3.2(0.7)$ & $3.1(0.8)$ \\
Gallop (Raw score 0-4) & $3.2(0.8)$ & $3.2(0.8)$ & $3.3(0.8)$ \\
Hop (Raw score 0-4) & $3.2(0.8)$ & $2.1(0.8)$ & $3.3(0.8)$ \\
Horizontal jump (Raw score 0-3) & $2.2(0.8)$ & $2.2(0.8)$ & $2.2(0.8)$ \\
Skip (Raw score 0-4) & $2.3(0.8)$ & $2.6(1.0)$ & $2.4(0.8)$ \\
Slide (Raw score 0-4) & $2.6(0.9)$ & $32.6(7.4)$ & $2.6(0.9)$ \\
Locomotor sub-test (Raw score 0-46) & $33.3(7.2)$ & $2.7(1.2)$ & $33.9(7.0)$ \\
Two-hand strike (Raw score 0-5) & $2.8(1.2)$ & $2.9(0.9)$ & $2.9(1.1)$ \\
One-hand strike (Raw score 0-4) & $2.9(0.9)$ & $1.8(0.9)$ & $2.9(0.9)$ \\
One-hand dribble (Raw score 0-3) & $1.8(0.9)$ & $2.1(0.8)$ & $1.8(0.9)$ \\
Catch (Raw score 0-3) & $2.1(0.8)$ & $2.6(1.0)$ & $2.1(0.9)$ \\
Kick (Raw score 0-4) & $2.7(1.0)$ & $2.9(0.9)$ & $2.8(1.0)$ \\
Over-hand throw (Raw score 0-4) & $2.9(0.9)$ & $3.0(0.9)$ & $2.9(1.0)$ \\
Under-hand throw (Raw score 0-4) & $3.0(0.9)$ & $36.0(8.4)$ & $3.0(0.9)$ \\
Ball skill sub-test (Raw score 0-54) & $36.3(8.6)$ & $68.6(13.4)$ & $36.5(8.7)$ \\
TGMD-3 total score (Raw score 0-100) & $69.5(13.5)$ & & $70.4(13.6)$ \\
\hline
\end{tabular}

TGMD-3 stands for the Test for Gross Motor Development $-3^{\text {rd }}$ Edition. 


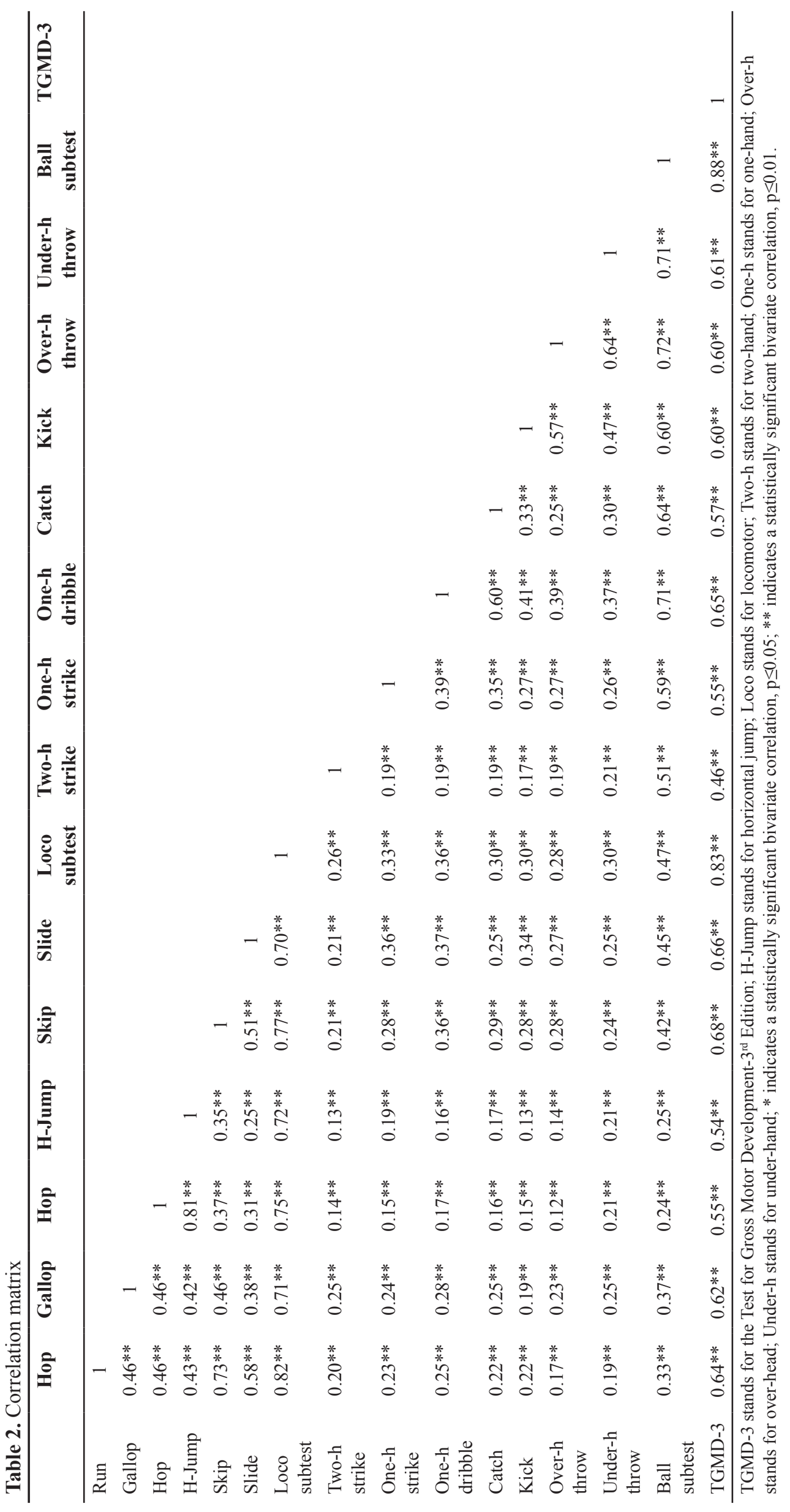


els of motor skill competence. These experiences including environment, physical education, SES, parental support, etc. Additionally, Venetsanou and Kambas (2010) also reported that family SES affected children's motor development. In this review study, they found in majority of relevant studies children of lower socioeconomic classes seem to perform worse than those of the middle/high classes in motor development. Consequently, a number of plausible reasons may explain the lower-class children's in the present study had poorer motor competence. Low SES children's motor incompetence may be associated with poor pre-and post-natal nutrition supplementation, which may have affected the central nervous system. In the present study, Low SES children in "Nevada Zoom" schools may not be encouraged to develop motor skills during school day, as they did not have physical education classes in regular school schedule. In contrast, high SES children had 2-3 physical education classes taught by certified physical educators each week, plus recess opportunities for physical activity in every school day. Therefore, high SES sample in this study may have plenty of opportunities for perceptual-motor experiences, which are beneficial to their motor skill development. In terms of the outof-school settings, low SES children living in disadvantage communities may suffer from the lack of facilities and space that prevents them from developing their motor skills (Eime et al., 2015; Kercood et al., 2015; Eime, et al., 2017). On the other hand, children from higher classes may have a greater number and variety of resources than children from lower classes.

There are a number of limitations to this study that must be considered before the results can be generalized. First, the sample consisted of children recruited across four schools from the Western U.S., specifically from a state characterized as having an arid climate; therefore the results are questionable if generalized to children belonging to other geographical regions. Second, the study design was observational and cross-sectional; therefore, no causal inferences can be made. Third, potential confounding not controlled for that may have influenced the results included physical activity behaviors and health-related fitness. Because of the bi-directional relationship between these constructs and gross motor competency, it is unknown whether the variability in TGMD-3 scores observed among grade levels and SES strata were partially because of variability in these behaviors and characteristics. Finally, as stated in the Methods, children from the high SES schools had physical education classes and children from lower SES schools did not; therefore, the differences in gross motor competency may have been due to the presence of physical education curricula, not the SES of the schools.

\section{CONCLUSION}

In conclusion, gross motor skill competency varied across grade levels and SES strata in a sample of young children from the Western U.S. The results of this study support previous work and clarify the potential relationship between gross motor competency and SES. Interestingly, sex did not mod- ify any of the observed relationships. Given the importance of gross motor competency and its relationship with healthy physical activity behaviors and consequent health-related fitness levels, exploring the factors relating to gross motor competency, such as grade level and SES, is imperative to improve the health of at-risk youth.

\section{Conflicts of Interest}

The author declares no conflict of interest.

\section{REFERENCES}

Ali, A., Pigou, D., Clarke, L., \& McLachlan, C. (2017). Literature review on motor skill and physical activity in preschool children in New Zealand. Advances in Physical Education, 7, 10-26.

Barnett, L. M., Ridgers, N. D., \& Salmon, J. (2015). Associations between young children's perceived and actual ball skill competence and physical activity. Journal of Science and Medicine in Sport, 18, 167-171.

Burns, R. D., Brusseau, T. A., Fu, Y., \& Hannon, J. C. (2015). Predictors and trends of motor skill performance in atrisk elementary school-aged children. Perceptual and Motor Skills, 121, 284-299.

Burns, R. D., Brusseau, T. A., Fu, Y., \& Hannon, J. C. (2017). Gross motor skills and cardiometabolic risk in children: A mediation analysis. Medicine \& Science in Sports \& Exercise, 49(4), 746-751.

Burton, A. W., \& Miller, D. E. (1998). Movement skill assessment. Champaign, IL: Human Kinetics.

Davis, W. E., \& Burton, A. W. (1991). Ecological task analysis: Translating movement behavior theory into practice. Adapted Physical Activity Quarterly, 8, 154-177.

Deflandre, A., Lorant, J., Gavarry, O., \& Falgairette, G. (2001). Determinants of physical activity and sports activities in French school children. Perceptual and Motor Skills, 92, 399-411.

Eime, R. M., Casey, M., Harvey, J. H., Sawyer, N. A., Symons, C. M., \& Payne, W. R. (2015). Socioecological factors potentially associated with participation in physical activity and sport: A longitudinal study of adolescent girls. Journal of Science and Medicine in Sport, 18, 684-690.

Eime, R. M., Harvey, J. H., Charity, M. J., Casey, M., Westerbeek, H., \& Payne, W. R. (2017). The relationship of sport participation to provision of sports facilities and socioeconomic status: a geographical analysis. Australian and New Zealand Journal of Public Health, 41, 248-255.

Fisher, A., Reilly, J. J., Kelly, L. A., Montgomery, C., Williamson, C., Paton, J. P., \& Grant S. (2005). Fundamental movement skills and habitual physical activity in young children. Medicine \& Science in Sports \& Exercise, 37, 684-688.

Goodway, J. D., \& Branta, C. F. (2003). Influence of a motor skill intervention on fundamental motor skill development of disadvantaged preschool children. Research Quarterly for Exercise and Sport, 74, 36-46.

Gesell, A. (1928). Infancy and human growth. New York: Macmillan. 
Goodway, J. D., \& Rudisill, M. E. (1997). Perceived physical competence and actual motor skill competence of African American preschool children. Adapted Physical Activity Quarterly, 14, 314-326.

Hardy, L. L., Reinten-Reynolds, T., Espinel, P., Zask, A., \& Okely A, D. (2012). Prevalence and correlates of low fundamental movement skill competency in children. Pediatrics, 130, 390-398.

Holfelder, B., \& Schott, N. (2014). Relationship of fundamental movement skills and physical activity in children and adolescents: A systematic review. Psychology of Sport and Exercise, 15, 382-391.

Kercood, S., Conway, T. L., Saelens, B. E., Frank, L. D., Cain, K. L., \& Sallis, J. F. (2015). Parent rules, barriers, and places for youth physical activity vary by neighborhood walkability and income. Children, Youth and Environments, 25, 100-118.

Lai, S. K., Costigan, S. A., Morgan, P. J., Luban, D. R., Stodden, D. F., Salmon, J., \& Barnett, L. M. (2014). Do school-based interventions focusing on physical activity, fitness, or fundamental movement skill competency produce a sustained impact in these outcomes in children and adolescents? A systematic review of follow-up studies. Sports Medicine, 44, 67-79.

Lampard, A. M., Jurkowski, J. M., Lawson, H. A., \& Davison, K. K. (2013). Family ecological predictors of physical activity parenting in low-income families. Behavioral Medicine, 39, 97-103.

Logan, S, W., Webster, E. K., Getchell, N., Pfeiffer, K. A., \& Robinson, L. E. (2015). Relationship between fundamental motor skill competence and physical activity during childhood and adolescence: a systematic review. Kinesiology Review, 4, 416-426.

Pangrazi, R. P., \& Beighle, A. (2013). Dynamic physical education for elementary school children. $17^{\text {th }} \mathrm{Ed}$. New York, NY: Pearson.
Payne, V.G., \& Isaacs, L.D. (2011). Human motor development: A lifespan approach, $8^{\text {th }}$ ed., McGraw-Hill, Boston, MA (2011)

Robinson, L. E., Rudsill, M. E., \& Goodway, J. (2009). Instructional climates in preschool children who are atrisk. Part II: Perceived physical competence. Research Quarterly for Exercise and Sport, 80, 543-551.

Robinson, L. E., Stodden, D. F., Barnett, L. M., Lopes, V. P., Logan, S. W., Rodrigues, L. P., \& D'Hondt, E. (2015). Motor competence and its effect on positive developmental trajectories of health. Sports Medicine, 45, 1273-1284.

Stodden, D. F., Gao, Z., Goodway, J. D., \& Langendorfer, S. J. (2014). Dynamic relationships between motor skill competence and health-related fitness in youth. Pediatric Exercise Science, 26, 231-241.

Stodden, D. F., Goodway, J. D., \& Langendorfer, S. J. (2008). A developmental perspective on the role of motor skill competence in physical activity: An emergent relationship. Quest, 60, 290-306.

Trudeau, F., \& Shephard, R. J. (2008). Physical education, school physical activity, school sports and academic performance. International Journal of Behavioral Nutrition and Physical Activity, 5, 10. doi:10.1186/1479-5868-5-10

Ulrich, D.A. (2000). Test of Gross Motor Development. $2^{\text {nd }}$ ed. Austin, TX: Pro-Ed, 1-5.

Venetsanou, F., \& Kambas, A. (2010). Environmental factors affecting preschoolers' motor development. Early Childhood Education Journal, 37, 319-327.

Webster, E. K., \& Ulrich, D. A. (2017). Evaluation of the psychometric properties of the test of gross motor development-third edition. Journal of Motor Learning and Development, 5, 45-58.

Williams, H. G., Pfeiffer, K. A., O’Neill, J. R., Dowda, M., Mclver, K. L., Brown, W. H., \& Pate, R. R. (2008). Motor skill performance and physical activity in preschool children. Obesity, 16, 1421-1426. 